\section{Information on co-morbidity on inpatient admissions}

Dear Editor - The HRB would be delighted to include information on co-morbidity on inpatient admissions as suggested by Dixit and Payne (Ir J Psych Med 2008, 25(4)). We would be happy to do so were secondary or accompanying diagnoses supplied to us as our forms/systems allow. Unfortunately clinicians seldom if ever return co-morbid diagnostic information; indeed it is at times difficult enough to acquire a principal or preliminary diagnosis. It is understandable that diagnosticians may have difficulty in complex presentations in establishing a definitive diagnosis at or shortly after admission and for this reason the facility for assigning a preliminary diagnosis on admission is provided. Where a preliminary diagnosis is later superseded there is accommodation on the forms for a final discharge diagnosis. The HRB is most anxious to progress information systems in mental health services as recommended in A Vision for Change and is in the process of significant developments in this field in collaboration with HSE and other stakeholders (www.hrb.ie).

We would be delighted to present and review with psychiatrists (consultants and trainees) our activities in this area. Accordingly we would be happy to make presentations to such bodies or groups as are thought relevant - through the Irish Post Graduate Training Committee or the College of Psychiatrists in Ireland. Antoinette Daly, Dermot Walsh, Health Research Board, Dublin 2.

\section{Antoinette Daly Research Officer Mental Health Research Unit Health Research Board An Bord Taighde Sláinte Knockmaun House 42-47 Lower Mount Street Dublin 2, Ireland.}

\section{Methadone and its effect on QTc prolongation}

Dear Editor - Methadone is a synthetic opioid which has been successful used in treating heroin addiction and chronic pain syndrome in palliative care for more than 30 years. Methadone maintenance programmes for heroin dependency has shown reduction in mortality and morbidity. It has shown direct reductions in addiction related crime and drug arrests. ${ }^{1-3}$ Several case reports have highlighted methadone's association with QT prolongation and the risk of Torsades de Pointes, a potentially lethal ventricular tachycardia. Levacetylmethadol, a derivative of methadone was withdrawn from US markets in 2003 due to prolongation of OT interval and an association with Torsades de Pointes. ${ }^{4-6}$

Methadone delays cardiac repolarisation by blocking the rapid component of $1 \mathrm{kr}$ potassium channels, encoded for by human ether a-go-go related gene (hERG) or $\mathrm{kCNH} 2$. Methadone is metabolised by isoforms CYP3A4 and to a lesser extent by the isoenzyme CYP 2D6 of the hepatic cytochrome-P450-system. Several risk factors have been identified as contributing to QT prolongation, including high methadone dose, structural or ischaemic heart disease, family history of sudden cardiac death, hypokalaemia, altered liver function and concurrent use of CYP3A4 inhibitors. Ingestion of cocaine and methadone can also contribute to OT prolongation. ${ }^{4,8}$

Patients on methadone maintenance programmes are therefore automatically at risk of OT prolongation due to frequency of concomitant medical conditions, cocaine abuse and prescription of additional medications. ${ }^{9}$

\section{Case No 1.}

$\mathrm{LH}$ is a 29 -year-old lady on the methadone maintenance programme for the past 10 years. She has a background history of ongoing heroin and cocaine addiction. She attends the drug treatment centre and is dispensed methadone $110 \mathrm{mg}$ daily. In addition, she has multiple medical problems including HIV, hepatitis $\mathrm{C}$, pneumonia, pericarditis and bilateral lower limb ulcers. She requires regular antibiotic treatment and is currently prescribed clarithromyan. She is treated for depression with Sertraline (SSRI.). LH presented to Accident and Emergency Department with fainting episodes, dizziness, shortness of breath, facial pallor and pyrexia. Her urine toxicology was positive for heroin, methadone and cocaine.

A 12 lead ECG showed QTc prolongation 540 msecs. She was treated successfully for a lower respiratory tract infection. Her methadone dose was gradually reduced from $110 \mathrm{mg}$ to $80 \mathrm{mg}$. Her ECG showed reduction of QTc prolongation to 410 msecs. She was discharged and referred back to drug treatment services.

\section{Case No 2.}

$\mathrm{ML}$ is a 28-year-old gentleman on the methadone maintenance programme for the last 8 years. He has a background of heroin and cocaine addiction and also admits to abusing methadone. $\mathrm{He}$ is dispensed methadone $120 \mathrm{mg}$ daily. His medical history includes HIV, hepatitis $\mathrm{C}$, pneumonia, chronic leg ulcers and deep vein thrombosis. He is prescribed fluoxetine for depression. He was prescribed ciprofloxacin for a lower respiratory tract infection. He presented to an Accident and Emergency department and was admitted medically with symptoms of chest pain, shortness of breath, cough, dizziness, tiredness and feeling faint. His urine toxicology was positive for heroin, methadone, cocaine and benzodiazepines. His 12 lead ECG showed QTc $490 \mathrm{msec}$. He was treated with co-amoxiclav for pneumonia. His methadone dose was reduced to $100 \mathrm{mg}$ once daily. His ECG then showed QTc 417 msec. He was discharged back to drug treatment services.

\section{Discussion}

Methadone induced QT prolongation is not an uncommon event and is associated with Torsades de Pointes, a lifethreatening ventricular arrhythmia. Patients on the methadone maintenance programmes may have a history of abusing cocaine, tend to have additional medical problems, may also be taking additional medications (eg. CYPL3A4 inhibitors) and are prone to experience electrolyte disturbances. These risk factors may act synergistically with methadone to prolong the QT interval. Heroin addicts sometimes faint whilst using recreational drugs and doctors might attribute these episodes of syncope to illicit drug use and thereby underestimate the incidence of Torsades de Pointes in this population. ${ }^{10,11}$ 